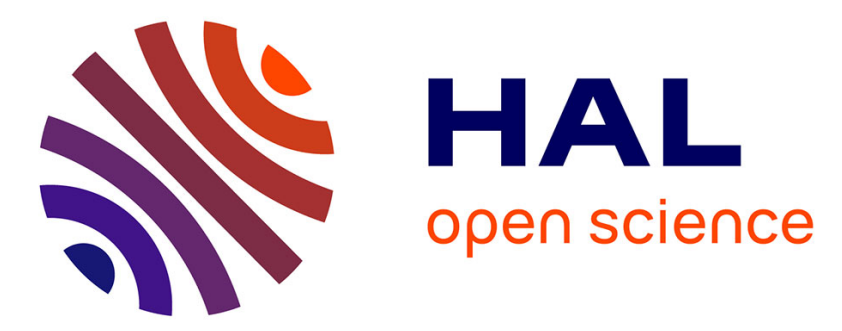

\title{
Reflection-based technique for synthesis of structured controllers with guaranteed local convergence
}

\author{
Mohamed Yagoubi, Redouane Chaibi
}

\section{To cite this version:}

Mohamed Yagoubi, Redouane Chaibi. Reflection-based technique for synthesis of structured controllers with guaranteed local convergence. International Journal of Systems Science, 2020, pp.1-12. 10.1080/00207721.2020.1817612 . hal-02937315

\section{HAL Id: hal-02937315 https://hal.science/hal-02937315}

Submitted on 11 Feb 2022

HAL is a multi-disciplinary open access archive for the deposit and dissemination of scientific research documents, whether they are published or not. The documents may come from teaching and research institutions in France or abroad, or from public or private research centers.
L'archive ouverte pluridisciplinaire HAL, est destinée au dépôt et à la diffusion de documents scientifiques de niveau recherche, publiés ou non, émanant des établissements d'enseignement et de recherche français ou étrangers, des laboratoires publics ou privés. 


\title{
Reflection-based technique for control synthesis under information structure constraints with guaranteed convergence
}

\author{
Mohamed Yagoubi ${ }^{\mathrm{a}}$ and Redouane Chaibi ${ }^{\mathrm{b}}$ \\ ${ }^{a}$ IMT Atlantique, LS2N, CNRS (UMR 6004), Nantes, France; bSidi-Mohamed Ben-Abdellah \\ University, Fès, Morocco.
}

\section{ARTICLE HISTORY}

Compiled May 5, 2020

\begin{abstract}
This paper deals with stabilization, $H_{2}$ and $H_{\infty}$ static feedback synthesis, under information structure constraints. The proposed approach relies on a prominent property of Hurwitz-stable matrices set and a Douglas-Rachford (DR) reflection method type for finding a point in the intersection of two closed sets. The method proposed in this paper emulates the genericity of the Linear Matrix Inequalities framework while keeping the feedback gain separated from any matrix with direct or indirect connection to the Lyapunov function. The link between the sequence of iterates generated by the proposed algorithm and a continuous dynamical system results in the use of Lyapunov stability theory for guaranteeing the convergence. Several examples are given to prove the validity of the proposed method.
\end{abstract}

\section{KEYWORDS}

Nearest matrix problems, Hurwitz-stable matrices set, Douglas-Rachford reflection method, output feedback control, information structure constraints.

\section{Introduction}

Several control problems can be recast as an optimization of a linear criteria under one or several LMI constraints (Boyd, El-Ghaoui, Feron \& Balakrishnan, 1994; VanAntwerp \& Braatz, 2000). The solution of the control problem is then derived efficiently using Semi-Definite Programming (SDP). However, not all control problems can be cast as convex optimization ones, especially when it comes to a control problem under information structure constraints (Rubio-Massegu, Rossell, Karimi \& PalaciosQuinonero, 2013) or a control problem involving various specifications such as the mixed $H_{2} / H_{\infty}$ control case (Scherer, Gahinet \& Chilali, 1997). The rise of LMIs since later 1990s have led to different strategies relaxing these problems. Unfortunately they were not suited for medium or large size problems. Due to the presence of Lyapunov variables, whose number grows quadratically with the system size, LMI programs (and hence BMI ones) (VanAntwerp \& Braatz, 2000) quickly lead to problem sizes where existing numerical algorithms (interior-point algorithm for convex cases, for instance, or cutting-plane algorithm for bilinear programs) fail (Lewis, 2007). These considerations are true in the case of state feedback and are even more exacerbated in the case

Email: mohamed.yagoubi@imt-atlantique.fr 
of static (with or without information constraints) output feedback design (see the survey paper (Sadabadi \& Peaucelle, 2016) and references therein).

In that context, it is still of interest to pave the way for an alternative solution that can be numerically competitive to existing methods and takes into account different control objectives as simply as for the Lyapunov/LMI paradigm. It must also keep a separation between the output (or state) feedback gain and any matrix with direct or indirect connection to the Lyapunov function.

The original approach, proposed in this paper, relies among others on DouglasRachford method which is a splitting method frequently employed for finding zeros of sums of maximally monotone operators. The success of the DR method (see the survey paper (Lindstrom \& Sims, 2018) and references therein) in the context of closed, convex, non-empty sets is well-known and understood from a theoretical view (Bauschke \& Moursi, 2017; Bauschke, Cruz, Nghia, Phan \& Wang, 2014; Bauschke, Combettes \& Luke, 2004). When one of the sets is non-convex the performance of such algorithm is still impressive (Borwein \& Sims, 2011). In particular, the proposed approach brings together wisely a prominent property of Hurwitz-stable matrices set and the DR iterates to propose a method for stabilization, $H_{2}$ and $H_{\infty}$ control synthesis, under information structure constraints. Besides, the proposed iterative method has a guaranteed convergence. The connection between the continuous limit sequence of the iterates and the trajectory of a continuous dynamical system results in the use of the direct method of Lyapunov stability theory to guarantee the local convergence.

The paper is organized as follows. Section 2 recalls some exiting and also introduces some new nearest matrix problems that are intensively used in the sequel. Section 3 proposes a new Douglas Rachford's like algorithm for stabilization, $H_{2}$ and $H_{\infty}$ control synthesis, under information structure constraints. Section 4 exhibits the convergence analysis and rate of the proposed iterative scheme. Section 5 illustrates the validity and efficiency of the proposed method through several numerical examples before concluding in section 6 .

Notations : Hereafter, $I_{n}$ designates the identity matrix of dimensions $n \times n$ and $\operatorname{vec}(Z)$ denotes the vec-operator that stacks the columns of $Z$ into a vector. $Z^{\top}$ denotes the transpose of a given real matrix $Z$ and $Z^{\dagger}$ denotes its Moore-Penrose pseudoinverse matrix. $\|Z\|=\left(\sum_{i, j} Z_{i j}^{2}\right)^{\frac{1}{2}}$ designate the Frobenius norm associated to the inner product $<Z_{1}, Z_{2}>=\operatorname{trace}\left(Z_{1}^{\top} Z_{2}\right)$. $Z>0(Z \geq)$ means that $Z$ is a positivedefinite (positive semi-definite) matrix. In the same vein, $Z<0(Z \leq)$ means that $Z$ is a negative-definite (negative semi-definite) matrix. The set of positive semi-definite (definite) matrices is denoted by $\mathbb{S}^{+}\left(\mathbb{S}^{+*}\right)$. In the same way, $\mathbb{S}_{g}^{-}\left(\mathbb{S}_{g}^{-*}\right)$ denotes the set of matrices with a negative semi-definite (definite) symmetric part. For a symmetric matrix represented block-wise, off-diagonal blocks are abbreviated with $\bullet$. Finally, without special stated, all matrices used in this paper are supposed to have appropriate dimensions.

\section{On some nearest matrix problems}

In this section, some preliminary definitions and results that will be used intensively in the sequel are presented. Hereafter, the Frobenius norm is chosen as a metric for its sub-multiplicative and invariance under rotations properties.

Definition 2.1 (Nearest matrix problem). A nearest matrix problem is defined as finding the nearest matrix $X_{F} \in \mathbb{D}^{m \times n}$ to a given matrix $Z \in \mathbb{R}^{m \times n}$ under Frobenius 
norm :

$$
X_{F}=\underset{X \in \mathbb{D}}{\arg \min }\|Z-X\|
$$

The solution of such problem depends entirely on the set $\mathbb{D}$ since $\|Z-X\|$ is a smooth function of $X$.

\subsection{Nearest matrix problem under LME constraint}

Let us consider the matrix set defined by :

$$
\mathbb{D}_{L} \triangleq\left\{X \in \mathbb{R}^{n \times n} / \exists \tilde{X} \in \mathbb{R}^{m \times p}: X=U_{0}+U_{1} \tilde{X} U_{2}\right\}
$$

with $U_{i}, i \in\{0,1,2\}$ are some given matrices of appropriate dimensions. Let us now define the nearest matrix problem under a Linear Matrix Equality (LME) constraint of the type $X=U_{0}+U_{1} \tilde{X} U_{2}$.

Definition 2.2 (Nearest matrix problem under LME). The nearest matrix problem under a Linear Matrix Equality (LME) is defined as :

$$
X_{F}=\underset{X \in \mathbb{D}_{L}}{\arg \min }\|Z-X\|
$$

Or equivalently,

$$
X_{F}=\underset{\tilde{X} \in \mathbb{R}^{m \times p}}{\arg \min }\left\|Z-\left(U_{0}+U_{1} \tilde{X} U_{2}\right)\right\| .
$$

This problem is convex, since it can be recast as a linear constrained Quadratic Program (QP), hence it can be solved efficiently. In fact, using the vec operator notations:

$$
x_{v} \triangleq \operatorname{vec}(X), z_{v} \triangleq \operatorname{vec}(Z)
$$

one can easily establish the equality given below :

$$
\|Z-X\|^{2}=z_{v}^{\top} z_{v}-2 z_{v}^{\top} x_{v}+x_{v}^{\top} x_{v}
$$

which is quadratic in $x_{v}$. Moreover, according to (Jovovic \& Malesevic, 2014), the LME :

$$
X=U_{0}+U_{1} \tilde{X} U_{2}
$$

is consistent if and only if, for some pseudo-inverses $U_{1}^{\dagger}, U_{2}^{\dagger}$ verifying $U_{1} U_{1}^{\dagger} U_{1}=U_{1}^{\dagger}$ and $U_{2} U_{2}^{\dagger} U_{2}=U_{2}^{\dagger}$, the following equality holds :

$$
U_{1} U_{1}^{\dagger}\left(X-U_{0}\right) U_{2}^{\dagger} U_{2}=\left(X-U_{0}\right)
$$


Hence, using vec operator properties we obtain :

$$
\left(U_{2}{ }^{\top} U_{2}{ }^{\top} \otimes U_{1} U_{1}^{\dagger}\right)\left(x_{v}-\operatorname{vec}\left(U_{0}\right)\right)=\left(x_{v}-\operatorname{vec}\left(U_{0}\right)\right)
$$

which is a linear constraint in $x_{v}$.

Remark 1. A zero-non zero structure on the matrix $\tilde{X}$ can be added to the nearest matrix problem under LME (2) using a linear matrix equality of the form : $\tilde{X} \odot \Delta=\tilde{X}$ (where $\odot$ denotes the element wise Hadamard product) with $\Delta \in \mathbb{R}^{m \times p}$ a matrix of either zero or one elements.

On another note, the projections on the set of positive definite matrices $\mathbb{S}^{+*}$ and generalized negative definite matrices $\mathbb{S}_{g}^{-*}$ are presented hereinafter.

\subsection{Nearest positive matrix problem}

Projection onto the cone of positive semi-definite matrices denoted $\mathbb{S}^{+}$, is equivalent to finding the positive semi-definite approximant using the Frobenius norm metric. Since $\mathbb{S}^{+}$is convex the approximant is unique and is equivalent to the problem, first introduced by Higham in (Higham, 1988), given below :

$$
X^{+}=\underset{X=X^{\top} \geq 0}{\arg \min }\|Z-X\|
$$

The solution of (3), for real matrices, is given by the following Theorem (Higham, 1988):

Theorem 2.3. Let $Z \in \mathbb{R}^{n \times n}$ be a given matrix and $Z_{s}=\left(Z+Z^{\top}\right) / 2, Z_{s s}=(Z-$ $\left.Z^{\top}\right) / 2$ be the symmetric and skew-symmetric parts of $Z$ respectively. Let $Z_{s}=U H$ be a polar decomposition of $Z_{s}$ with $\left(U^{\top} U=I_{n}, H=H^{\top} \geq 0\right)$, then $X^{+}=\left(Z_{s}+H\right) / 2$ is the unique positive semi-definite approximant of $Z$ in the Frobenius norm.

Proof. Let $X$ be a symmetric positive semi-definite matrix.

$$
\|Z-X\|^{2}=\left\|Z_{s}-X\right\|^{2}+\left\|Z_{s s}\right\|^{2}+2<Z_{s}-X, Z_{s s}>
$$

This equality reduces to $\|Z-X\|^{2}=\left\|Z_{s}-X\right\|^{2}+\left\|Z_{s s}\right\|^{2}$ since $Z_{s s}$ is a skew-symmetric matrix. Hence, the problem consists in approximating $Z_{s}$. Let $Z_{s}=T \Lambda T^{\top}$ be a spectral decomposition with $T^{\top} T=I_{n}, \Lambda=\operatorname{diag}\left(\lambda_{i}\right)$, let $Y=T^{\top} X T$. Since the Frobenius norm is invariant by rotations we obtain :

$$
\begin{aligned}
\left\|Z_{s}-X\right\|^{2} & =\|\Lambda-Y\|^{2}=\sum_{i \neq j} y_{i j}^{2}+\sum_{i}\left(\lambda_{i}-y_{i i}\right)^{2} \\
& \geq \sum_{\lambda_{i}<0}\left(\lambda_{i}-y_{i i}\right)^{2}>\sum_{\lambda_{i}<0} \lambda_{i}^{2}
\end{aligned}
$$

Since $Y$ is positive semi-definite, the lower bound $\sum_{\lambda_{i}<0} \lambda_{i}^{2}$ is reached uniquely for the 
matrix $Y=\operatorname{diag}\left(d_{i}\right)$ where :

$$
d_{i}=\left\{\begin{array}{cc}
\lambda_{i}, & \lambda_{i} \geq 0 \\
0, & \lambda_{i}<0
\end{array}\right.
$$

that is : $X_{F}=T \operatorname{diag}\left(d_{i}\right) T^{\top}$. Finally, since $H=T \operatorname{diag}\left(\left|\lambda_{i}\right|\right) T^{\top}$, we get $X^{+}=\left(Z_{s}+\right.$ $H) / 2 \in \mathbb{S}^{+}$(Higham, 1988).

Remark 2. Projection on $\mathbb{S}^{+*}$ can be obtained in the same manner as in the last Theorem by substituting the equation (5) by the following equation

$$
d_{i}=\left\{\begin{array}{l}
\lambda_{i}, \lambda_{i}>0 \\
\epsilon, \quad \lambda_{i} \leq 0, \epsilon>0
\end{array}\right.
$$

with $\epsilon$ a sufficiently small positive real scalar (compared to the condition number associated to $Z_{s}$ ). This projection can be numerically obtained based on the Schur decomposition, for instance, using the spectral decomposition $Z_{s}=T \Lambda T^{\top}$ where $T$ is an orthogonal matrix and $\Lambda=\operatorname{diag}\left(\lambda_{i}\right), i=1,2, \ldots, n$. Then, $Z_{s}^{+}=T \bar{\Lambda} T^{T}$ where $\bar{\Lambda}=\operatorname{diag}\left(\bar{\lambda}_{i}^{+}\right)=\max \left\{\epsilon ; \lambda_{i}\right\}$ for $i=1,2, \ldots, n$.

\subsection{Nearest generalized negative matrix problem}

Unlike the nearest positive matrix problem solved by Higham, the following generalization is original and introduced for the first time in this paper. Let us consider the following set $\mathbb{S}_{g}^{-}=\left\{Z \in \mathbb{R}^{n \times n} / Z_{s}=\left(Z+Z^{\top}\right) / 2 \leq 0\right\}$ including matrices with a negative semi-definite symmetric part. Then we have $\mathbb{S}^{-} \subset \mathbb{S}_{g}^{-}$and projecting onto $\mathbb{S}_{g}^{-}$ can be seen as a generalization of the problem given in (3) and is given by :

$$
X_{g}=\underset{X \in \mathbb{S}_{g}^{-}}{\arg \min }\|Z-X\|
$$

if one consider $X_{s}=\left(X+X^{\top}\right) / 2, X_{s s}=\left(X-X^{\top}\right) / 2$ and $Z_{s}=\left(Z+Z^{\top}\right) / 2, Z_{s s}=$ $\left(Z-Z^{\top}\right) / 2$ the symmetric and skew-symmetric parts of $X$ and $Z$ respectively, then the function to be minimized in (7) becomes:

$$
\|Z-X\|^{2}=\left\|Z_{s}+Z_{s s}-X_{s}-X_{s s}\right\|^{2}
$$

which leads to

$$
\|Z-X\|^{2}=\left\|Z_{s s}-X_{s s}\right\|^{2}+\left\|Z_{s}-X_{s}\right\|^{2}+2<Z_{s s}-X_{s s}, Z_{s}-X_{s}>.
$$

This reduces to : $\|Z-X\|^{2}=\left\|Z_{s s}-X_{s s}\right\|^{2}+\left\|Z_{s}-X_{s}\right\|^{2}$ since $Z_{s s}-X_{s s}$ is skewsymmetric. Hence (7) can be obtained by solving the following minimization problem:

$$
\min _{X_{s s}=-X_{s s}^{\top}, X_{s} \in \mathbb{S}^{-}}\left\|Z_{s s}-X_{s s}\right\|^{2}+\left\|Z_{s}-X_{s}\right\|^{2}
$$


Since both $Z_{s s}$ and $X_{s s}$ are skew-symmetric matrices, the solution of (7) can be obtained by solving the following minimization problem:

$$
X_{g}=\underset{X_{s}=X_{s}^{T} \leq 0}{\arg \min }\left\|Z_{s}-X_{s}\right\|
$$

which is similar to problem (3). Finally, the solution of the nearest generalized negative matrix problem (7) is given by $X_{g}=Z_{s s}+X_{s}{ }^{-}$with $X_{s}{ }^{-}$is the projection of $Z_{s}$ onto $\mathbb{S}^{-*}$.

\section{Reflection based techniques for constrained control synthesis}

\subsection{Hurwitz-stable matrices set}

The set of Hurwitz-stable matrices, henceforth denoted by $\mathcal{H}_{s}$, is a simply connected open cone (Duan \& Patton, 1998) since it is the product of two convex open cones. It follows from that, as shown in (Duan \& Patton, 1998), any Hurwitz-stable matrix $Z$ can be expressed as a product of a generalized negative definite matrix $X \in \mathbb{S}_{g}^{-*}$ and a positive definite matrix $Y \in \mathbb{S}^{+*}$ as stated by the following Theorem.

Theorem 3.1. (Duan \& Patton, 1998) A matrix $Z \in \mathbb{R}^{n \times n}$ is Hurwitz-stable if and only if there exist a generalized negative definite matrix $X$ and positive definite matrix $Y$ such that : $Z=X Y$.

\subsection{Nearest Hurwitz-stable matrix problem}

The projection of a given matrix $Z_{0} \in \mathbb{R}^{n \times n}$ onto $\mathcal{H}_{s}$ is yet another nearest matrix problem given by the following expression :

$$
Z_{F}=\underset{Z \in \mathcal{H}_{s}}{\arg \min }\left\|Z_{0}-Z\right\|
$$

Using the result of Theorem 3.1, one can recast this nearest matrix problem as the following original optimization problem :

$$
\min _{X \in \mathbb{S}_{g}^{-*}, Y \in \mathbb{S}^{+*}}\left\|Z_{0}-X Y\right\|
$$

To solve this problem a gradient-projection scheme is proposed and presented by iterations of the form :

$$
\left\{\begin{array}{c}
X_{k+1}=\left(X_{k}-\delta_{X} \partial_{X}\right)_{g}^{-} \\
Y_{k+1}=\left(Y_{k}-\delta_{Y} \partial_{Y}\right)^{+}
\end{array}\right.
$$

where $(\cdot)_{g}^{-},(\cdot)^{+}$designate the projections on $\mathbb{S}_{g}^{-*}$ and $\mathbb{S}^{+*}$ respectively. $\partial_{X}$ and $\partial_{Y}$ denote the sub-gradients given by :

$$
\left\{\begin{array}{l}
\partial_{X}=\left(Z_{0}-X_{k} Y_{k}\right) Y_{k}^{\top} \\
\partial_{Y}=X_{k}^{\top}\left(Z_{0}-X_{k} Y_{k}\right)
\end{array}\right.
$$


$\delta_{X}$ and $\delta_{Y}$ designate the adaptive step-sizes given by :

$$
\left\{\begin{array}{l}
\delta_{X}=\left\|\partial_{X}\right\|^{2} /\left\|\partial_{X} Y_{k}\right\|^{2} \\
\delta_{Y}=\left\|\partial_{Y}\right\|^{2} /\left\|X_{k} \partial_{Y}\right\|^{2}
\end{array}\right.
$$

Moreover, the initial starting points $X_{0}$ and $Y_{0}$ are computed using polar decomposition of $Z_{0}=U H$ that is : $X_{0}=(U)_{g}^{-}, Y_{0}=H$.

\subsection{Douglas-Rachford like technique for constrained control synthesis}

Douglas-Rachford methods (DR) are frequently used to find a feasible point (i.e. a point in the intersection) of two closed constraint sets in a Hilbert space. In our case, let us consider the following closed subsets ${ }^{1} \overline{\mathbb{D}}_{L} \subset \mathbb{D}_{L}$ and $\overline{\mathcal{H}}_{s} \subset \mathcal{H}_{s}$. The stabilization problem under information structure constraint for the linear time-invariant (LTI) system described by :

$$
\begin{cases}\dot{x}=A x+B u, & x \in \mathbb{R}^{n_{x}}, u \in \mathbb{R}^{n_{u}} \\ y=C x, & y \in \mathbb{R}^{n_{y}}\end{cases}
$$

can be recast as the following original feasibility problem :

$$
\text { Find } K \in \mathbb{R}^{n_{u} \times n_{y}} / Z \triangleq(A+B K C) \in \overline{\mathbb{D}}_{L} \cap \overline{\mathcal{H}}_{s}
$$

In order to simplify both notation and implementation the projections on each subset, which are no other than the nearest matrix problem under LME associated to $\overline{\mathbb{D}}_{L}$ and the nearest Hurwitz-stable matrix problem associated to $\overline{\mathcal{H}}_{s}$, will be noted $\mathcal{P}_{\overline{\mathbb{D}}_{L}}$ and $\mathcal{P}_{\overline{\mathcal{H}}_{s}}$ respectively, in the sequel. Hence, the corresponding reflection mappings noted $\mathcal{R}_{\overline{\mathbb{D}}_{L}}, \mathcal{R}_{\overline{\mathcal{H}}_{s}}$ are defined by :

$$
\mathcal{R}_{\overline{\mathbb{D}}_{L}} \triangleq \mu_{d} \mathcal{P}_{\overline{\mathbb{D}}_{L}}+\left(1-\mu_{d}\right) I, \mathcal{R}_{\overline{\mathcal{H}}_{s}} \triangleq \mu_{h} \mathcal{P}_{\overline{\mathcal{H}}_{s}}+\left(1-\mu_{h}\right) I
$$

with $\mu_{d} \in(0,2], \mu_{h} \in(0,2]$. In (Fält \& Giselsson, 2017), an optimal value of the adaptation parameters $\mu_{d}, \mu_{h}$ is found in the case of finding a point in the intersection of two (convex) affine subspaces. Let us just remind that the problem tackled with involves a convex and a potentially non-convex set. The (local) convergence, the smoothing effect of the proposed optimization scheme are discussed in the next section. Moreover, DR method is often referred to as reflect-reflect-average algorithm. See survey papers (Lindstrom \& Sims, 2018; Ryu \& Boyd, 2016) and references therein) that trace back its origin to the mid-1950s. It admits many variations and has proven its superiority on alternating projections algorithms. In this paper, an original DR-like scheme is proposed and is defined hereafter.

Definition 3.2 (Modified DR method (MDR)). Given an initial matrix $K_{0} \in \mathbb{R}^{n_{u} \times n_{y}}$, the MDR method generates a sequence $\left(K_{k}\right)_{k=1}^{\infty}$ as follows :

$$
\begin{gathered}
K_{k+1} \in T_{\overline{\mathbb{D}}_{L}, \overline{\mathcal{H}}_{s}}\left(K_{k}\right), \\
T_{\overline{\mathbb{D}}_{L}, \overline{\mathcal{H}}_{s}} \triangleq(1-\mu) I+\mu \mathcal{R}_{\overline{\mathbb{D}}_{L}} \mathcal{R}_{\overline{\mathcal{H}}_{s}}
\end{gathered}
$$

\footnotetext{
${ }^{1}$ An element wise linear constraint can be added, without loss of generality, to ensure the closedness of the considered subsets.
} 
$\mu \in(0,1]$ is some adaptation parameter.

\section{4. $\mathrm{H}_{\infty}$ and $\mathrm{H}_{2}$ characterizations}

In light of the stability characterization, original characterizations of $H_{\infty}$ and $\mathrm{H}_{2}$ performances are proposed hereafter. For this aim, let us consider the LTI system described by :

$$
\left\{\begin{array}{lll}
\dot{x}=A x+B_{w} w+B u, & & x \in \mathbb{R}^{n_{x}}, w \in \mathbb{R}^{n_{w}}, u \in \mathbb{R}^{n_{u}} \\
z=C_{z} x+D_{z} u, & & z \in \mathbb{R}^{n_{z}} \\
y=C x, & y \in \mathbb{R}^{n_{y}} . &
\end{array}\right.
$$

The following result presents the new $H_{\infty}$ performance characterization of the closedloop given by :

$$
\begin{cases}\dot{x}=A_{c} x+B_{w} w, & A_{c} \triangleq A+B K C \\ z=C_{c} x, & C_{c} \triangleq C_{z}+D_{z} K C\end{cases}
$$

Theorem 3.3. The closed-loop system given by (19) has an $H_{\infty}$ norm less than $\gamma>0$ if and only if there exist matrices $X \in \mathbb{R}^{n_{x} \times n_{x}}, Y \in \mathbb{R}^{n_{x} \times n_{x}}$ and $G \in \mathbb{R}^{n_{z} \times n_{x}}$ such that:

$$
\left[\begin{array}{c}
A_{c} \\
C_{c}
\end{array}\right]=\left[\begin{array}{c}
A \\
C_{z}
\end{array}\right]+\left[\begin{array}{c}
B \\
D_{z}
\end{array}\right] K C=\left[\begin{array}{c}
X \\
G
\end{array}\right] Y
$$

with $\left[\begin{array}{c}X \\ G\end{array}\right] V_{1}+V_{2} \in \mathbb{S}_{g}^{-*}$ is a generalized definite negative matrix and $Y \in \mathbb{S}^{+*}$ a definite positive matrix. $V_{1}$ and $V_{2}$ are some constant matrices given by:

$$
V_{1}=\left[\begin{array}{ll}
I_{n_{x}} & 0_{n_{x} \times n_{y}}
\end{array}\right], V_{2}=\frac{1}{2}\left[\begin{array}{cc}
\frac{1}{\gamma^{2}} B_{w} B_{w}^{\top} & 0 \\
0 & -I_{n_{y}}
\end{array}\right] .
$$

Proof. According to the bounded real-lemma, system (19) has an $H_{\infty}$ norm less than $\gamma>0$ if and only if there exist a matrix $Q=Q^{\top}>0$ such that :

$$
\left[\begin{array}{cc}
A_{c}^{\top} Q+Q A_{c}+C_{c}^{\top} C_{c} & \bullet \\
B_{1}^{\top} Q & -\gamma^{2} I
\end{array}\right]<0
$$

Since $A_{c}$ is Hurwitz-stable :

$$
\exists X \in \mathbb{S}_{g}^{-*}, Y=Q \in \mathbb{S}^{+*} / A c=X Y
$$

Moreover, $Y=Q$ is non singular means that :

$$
\exists G \in \mathbb{R}^{n_{y} \times n_{x}} / C_{c}=G Y
$$


Hence, the bounded real-lemma writes :

$$
Y\left(X^{\top}+X+N^{\top} N+\frac{1}{\gamma^{2}} B_{1} B_{1}^{\top}\right) Y<0
$$

which, by Schur's lemma, leads to

$$
\left[\begin{array}{cc}
X^{\top}+X+\frac{1}{\gamma^{2}} B_{1} B_{1}^{\top} & \bullet \\
G & -I
\end{array}\right]<0
$$

or equivalently,

$$
\left[\begin{array}{c}
X \\
G
\end{array}\right] V_{1}+V_{2} \in \mathbb{S}_{g}^{-*}
$$

Hence, the $H_{\infty}$ synthesis problem under information structure constraint for the LTI system given by (18) can be recast as the following feasibility problem :

$$
\text { Find } K \in \mathbb{R}^{n_{u} \times n_{y}} /\left[\begin{array}{l}
A_{c} \\
C_{c}
\end{array}\right] \in \overline{\mathbb{D}}_{L} \cap \overline{\mathcal{H}}_{\infty}
$$

with $\overline{\mathcal{H}}_{\infty}$ is the closed subset of matrices $Z$ that can be written as $\left[\begin{array}{c}A_{c} \\ C_{c}\end{array}\right]=\left[\begin{array}{c}A \\ C_{z}\end{array}\right]+$ $\left[\begin{array}{c}B \\ D_{z}\end{array}\right] K C=\left[\begin{array}{c}X \\ G\end{array}\right] Y$ with $\left[\begin{array}{c}X \\ G\end{array}\right] V_{1}+V_{2} \in \mathbb{S}_{g}^{-*}$ is a generalized definite negative matrix and $Y \in \mathbb{S}^{+*}$ a definite positive matrix.

Remark 3. This problem can be solved by the MDR like method $\left(T_{\overline{\mathbb{D}}_{L}, \overline{\mathcal{H}}_{\infty}}^{m}\right)$ as presented in Definition 3.2. The projections and reflections can be derived by gradientprojection algorithms as for the stability case.

In the same vain, the $H_{2}$ performance is presented hereafter.

Theorem 3.4. The closed-loop system given by (19) has an $\mathrm{H}_{2}$ norm less than $\gamma_{2}>0$ if and only if there exist matrices $X \in \mathbb{R}^{n_{x} \times n_{x}}, Y \in \mathbb{R}^{n_{x} \times n_{x}}$ and $N \in \mathbb{R}^{n_{z} \times n_{x}}$ such that :

$$
\left[\begin{array}{c}
A_{c} \\
C_{c}
\end{array}\right]=\left[\begin{array}{c}
A \\
C_{z}
\end{array}\right]+\left[\begin{array}{c}
B \\
D_{z}
\end{array}\right] K C=\left[\begin{array}{c}
X \\
G
\end{array}\right] Y
$$

with $\left[\begin{array}{l}X \\ G\end{array}\right] V_{1}+V_{3} \in \mathbb{S}_{g}^{-*}$ is a generalized definite negative matrix and $Y \in \mathbb{S}^{+*}$ a definite positive matrix verifying in addition the LME constraint trace $\left(B_{1}^{\top} Y B_{1}\right)<\gamma_{2}^{2}$. $V_{1}$ and $V_{3}$ are some constant matrices given by:

$V_{1}=\left[\begin{array}{cc}I_{n_{x}} & 0_{n_{x} \times n_{y}}\end{array}\right], V_{3}=\frac{1}{2}\left[\begin{array}{cc}0 & 0 \\ 0 & -I_{n_{y}}\end{array}\right]$. 


\section{Local Convergence Analysis}

Let us first recall that the proximal mapping (or prox-operator) of a convex function $f: \mathbb{R}^{n} \rightarrow \mathbb{R}$ is defined as :

$$
\operatorname{prox}_{f}(v)=\underset{x}{\arg \min }\left(f(x)+\frac{1}{2}\|x-v\|^{2}\right)
$$

For instance, if $f(x)=0, \operatorname{prox}_{f}(v)=v$. Moreover, if $f$ is the indicator function a of closed convex set $\mathbb{F}$, then $\operatorname{prox}_{f}$ is non other than the projection on $\mathbb{F}$ given by:

$$
\operatorname{prox}_{f}(v)=\underset{z \in \mathbb{F}}{\arg \min }\|z-v\|^{2}=\mathcal{P}_{\mathbb{F}}(v) .
$$

Recall also that if $f$ is convex, the $\operatorname{prox}_{f}(v)$ exists and is unique for all $v$. Another important point that should be recalled is that the Moreau-Yosida regularization (known as Moreau envelope) of a function $f$, (Moreau, 1965), is defined as :

$$
f_{m}(v)=\inf _{z}\left(f(z)+\frac{1}{2 \lambda}\|z-v\|^{2}\right), \quad \lambda>0
$$

Or equivalently,

$$
f_{m}(v)=f\left(\operatorname{prox}_{\lambda f}(v)\right)+\frac{1}{2 \lambda}\left\|\operatorname{prox}_{\lambda f}(v)-v\right\|^{2} .
$$

The Moreau envelope is usually used as an approximation of $f$, although regularization was not the purpose of the seminal paper (Moreau, 1965). Recall that in the nonsmooth case, it consists on a smooth regularization since it is always convex and its gradient $\nabla f_{m}$ exists and is given by :

$$
\nabla f_{m}(v)=\frac{1}{\lambda}\left(v-\operatorname{prox}_{\lambda f}(v)\right)
$$

Moreover, $\nabla f_{m}$ is Lipschitz continuous with constant $\frac{1}{\lambda}$.

In the MDR based technique context, we suppose that $\overline{\mathbb{D}}_{L}$ admits a quadratic indicator function $: z=\operatorname{vec}(Z) \rightarrow d(z)$ and $\overline{\mathcal{H}}$, which represents henceforth either $\overline{\mathcal{H}}_{s}$, $\overline{\mathcal{H}}_{\infty}^{m}$ or $\overline{\mathcal{H}}_{2}^{m}$, admits a non-smooth indicator function $: z=\operatorname{vec}(Z) \rightarrow h(z)$. Given an initial point $z_{0}$ (derived from a randomly chosen tri-diagonal matrix $Z_{0} \in \mathbb{R}^{n \times n}$ for instance representing the initial closed-loop matrix $A_{c}$ ), the MDR method generates a sequence $\left(z_{k}\right)_{k=1}^{\infty}$ as follows :

$$
z_{k+1}=\left((1-\mu) I+\mu \mathcal{R}_{\overline{\mathcal{H}}} \mathcal{R}_{\overline{\mathbb{D}}_{L}}\right)\left(z_{k}\right), \quad \mu \in(0,1]
$$

where the mappings noted $\mathcal{R}_{\overline{\mathbb{D}}_{L}}, \mathcal{R}_{\overline{\mathcal{H}}}$ are defined by :

$$
\begin{aligned}
& \mathcal{R}_{\overline{\mathbb{D}}_{L}} \triangleq \mu_{d} \mathcal{P}_{\overline{\mathbb{D}}_{L}}+\left(1-\mu_{d}\right) I, \quad \mu_{d} \in(0,2] \\
& \mathcal{R}_{\overline{\mathcal{H}}} \triangleq \mu_{h} \mathcal{P}_{\overline{\mathcal{H}}}+\left(1-\mu_{h}\right) I, \quad \mu_{h} \in(0,2]
\end{aligned}
$$

Hence, the problem tackled with, in this section, consists in analysing the convergence and the convergence rate of the MDR search scheme given by equations (31), 
(32) and (33) by means of a Lyapunov based stability analysis of a dynamical system whose trajectory goes through the discrete iterates $\left(z_{k}\right)$. It is shown, hereafter, that the MDR iterates can be interpreted as a gradient-descent method for minimizing a differentiable function, subsequently constructed, namely $f_{m d r}$. In that case, given an initial point $z_{0} \in \mathbb{R}^{n^{2}}$, the MDR method generates a sequence $\left(z_{k}\right)_{k=1}^{\infty}$ that is a discretization of the trajectory of the continuous dynamical system given by

$$
\dot{z}(t)=\mu\left(\mathcal{R}_{\overline{\mathcal{H}}} \mathcal{R}_{\overline{\mathbb{D}}_{L}}-I\right) z(t), \quad z\left(t_{0}\right)=z_{0}
$$

using the forward discretization $\dot{z}(t) \approx z_{k+1}-z_{k}$ and $z(t) \approx z_{k}$ approximation (for simplicity of presentation, the sample time is fixed to 1 without loss of generality). Therefore, we aim here to recast (34) as

$$
\dot{z}(t)=-\nabla f_{m d r}(z), \quad z\left(t_{0}\right)=z_{0}
$$

According to (30) one can rewrite the reflections $\mathcal{R}_{\overline{\mathbb{D}}_{L}}, \mathcal{R}_{\overline{\mathcal{H}}}$ as follows :

$$
\mathcal{R}_{\overline{\mathbb{D}}_{L}}(z)=\left(I-\mu_{d} \nabla d_{m}\right)(z), \quad \mathcal{R}_{\overline{\mathcal{H}}}(z)=\left(I-\mu_{h} \nabla h_{m}\right)(v) .
$$

This leads to :

$$
\mu\left(I-\mathcal{R}_{\overline{\mathcal{H}}} \mathcal{R}_{\overline{\mathbb{D}}_{L}}\right)(z)=\mu \mu_{d} \nabla d_{m}(z)+\mu \mu_{h} \nabla h_{m}\left(z-\mu_{d} \nabla d_{m}(z)\right)
$$

Since, $d$ is twice continuously differentiable the term $\left(I-\frac{\mu_{d}}{2} \nabla^{2} d_{m}(z)\right)$ exists. Besides, since $d$ is quadratic the term $\left(I-\frac{\mu_{d}}{2} \nabla^{2} d_{m}(z)\right)$ is constant that is $\left(I-\frac{\mu_{d}}{2} \nabla^{2} d_{m}(z)\right) \triangleq \Gamma$. At this point we are able to construct $f_{m d r}$ as follows :

$$
f_{m d r}(z) \triangleq \mu\left(\Gamma^{-1}\left(\mu_{d} d_{m}(z)+\mu_{h} h_{m}\left(z-\mu_{d} \nabla d_{m}(z)\right)-\frac{\mu_{d}}{2}\left(\nabla d_{m}(z)\right)^{2}\right)\right)
$$

Hence,

$$
\dot{z}(t)=\mu\left(\mathcal{R}_{\overline{\mathcal{H}}} \mathcal{R}_{\overline{\mathbb{D}}_{L}}-I\right) z(t)=-\nabla f_{m d r}(z) .
$$

The asymptotic stability of a point $z^{*}$ supposed to be a strict local minimizer of $f_{m d r}$, follows from Lyapunov theorem by choosing the following Lyapunov function :

$$
V_{L}(z) \triangleq f_{m d r}(z)-f_{m d r}\left(z^{*}\right)
$$

Then, one can note that

$$
\dot{V}_{L}(z)=<\nabla f_{m d r}(z), \dot{z}>=-\left\|\nabla f_{m d r}(z)\right\|^{2} \leq 0 .
$$

Assuming that $z^{*}$ is an isolated local minimizer then $\nabla f_{m d r}(z) \neq 0$. Therefore, $z^{*}$ is an asymptotically stable equilibrium point of (35) and consequently for the continuoustime dynamical system (34).

Furthermore, choosing a Lyapunov function of the form :

$$
V_{L}(z, t) \triangleq t\left(f_{m d r}(z)-f_{m d r}\left(z^{*}\right)\right)+\frac{1}{2}\left\|z-z^{*}\right\|^{2}, \forall t \geq 0 .
$$


leads to

$$
f_{m d r}(z)-f_{m d r}\left(z^{*}\right) \leq \frac{1}{t} V_{L}\left(z_{0}, t_{0}\right)
$$

which means that the convergence rate is of $O\left(\frac{1}{t}\right)$. The last inequality writes also :

$$
f_{m d r}(z)-f_{m d r}\left(z^{*}\right) \leq \frac{1}{t} c\left(\mu, \mu_{d}, \mu_{h}\right)
$$

where $c$ depends only on parameters $\mu, \mu_{d}, \mu_{h}$ since $z_{0}$ is fixed.

Remark 4. Inequality (42) means that the adaptation parameters have a direct effect, as expected, on the convergence rate.

\section{Numerical Examples}

\subsection{Stabilization}

An academic example borrowed from (Rubio-Massegu et al., 2013) consisting in a LTI system given by (14) with the following state-space matrices data :

$$
A=\left[\begin{array}{ccccc}
-4 & 0 & -2 & 0 & 0 \\
0 & -2 & 0 & 2 & 0 \\
0 & 0 & -2 & 0 & -1 \\
0 & -2 & 0 & -1 & 0 \\
3 & 0 & -2 & 0 & -1
\end{array}\right], B=\left[\begin{array}{ccc}
1 & 0 & 0 \\
1 & 0 & 0 \\
0 & 0 & 0 \\
0 & 1 & 0 \\
0 & 0 & 1
\end{array}\right], C=\left[\begin{array}{ccccc}
1 & 0 & 0 & 0 & 0 \\
0 & 1 & 0 & 0 & 0 \\
0 & 0 & 0 & 0 & 1
\end{array}\right]
$$

is considered to test the MDR method (with $\mu=\frac{1}{2}, \mu_{d}=\mu_{h}=2$ ) for static output feedback stabilization. Starting from $K_{0}=0_{3 \times 3}$ with $\sigma(A)=\{-1.5000 \pm 1.9365 i,-3.7004 \pm$ $1.1286 i, 0.4009\}$, only 2 iterations ${ }^{2}$ and a computation-time less than 1 second to obtain the stabilizing static output feedback $K^{*}$ given by :

$$
K^{*}=\left[\begin{array}{ccc}
-0.0526 & 0.0167 & -0.2446 \\
0.0725 & -0.0465 & 0.1227 \\
-0.3732 & 0.0956 & -0.7068
\end{array}\right]
$$

with $\sigma\left(A+B K^{*} C\right)=\{-3.8230 \pm 1.2702 i,-1.4944 \pm 1.9661 i,-0.1079\}$. When an additional structure constraint, of the type $\tilde{K} \odot \Delta=\tilde{K}$, with

$$
\Delta=\left[\begin{array}{lll}
1 & 0 & 1 \\
1 & 0 & 1 \\
1 & 0 & 1
\end{array}\right]
$$

${ }^{2}$ On Matlab@Mathworks, Processor Intel Core i7-4500U CPU @ 1.8GHz 
Table 1. MDR method applied to static output feedback problems from COMPleib

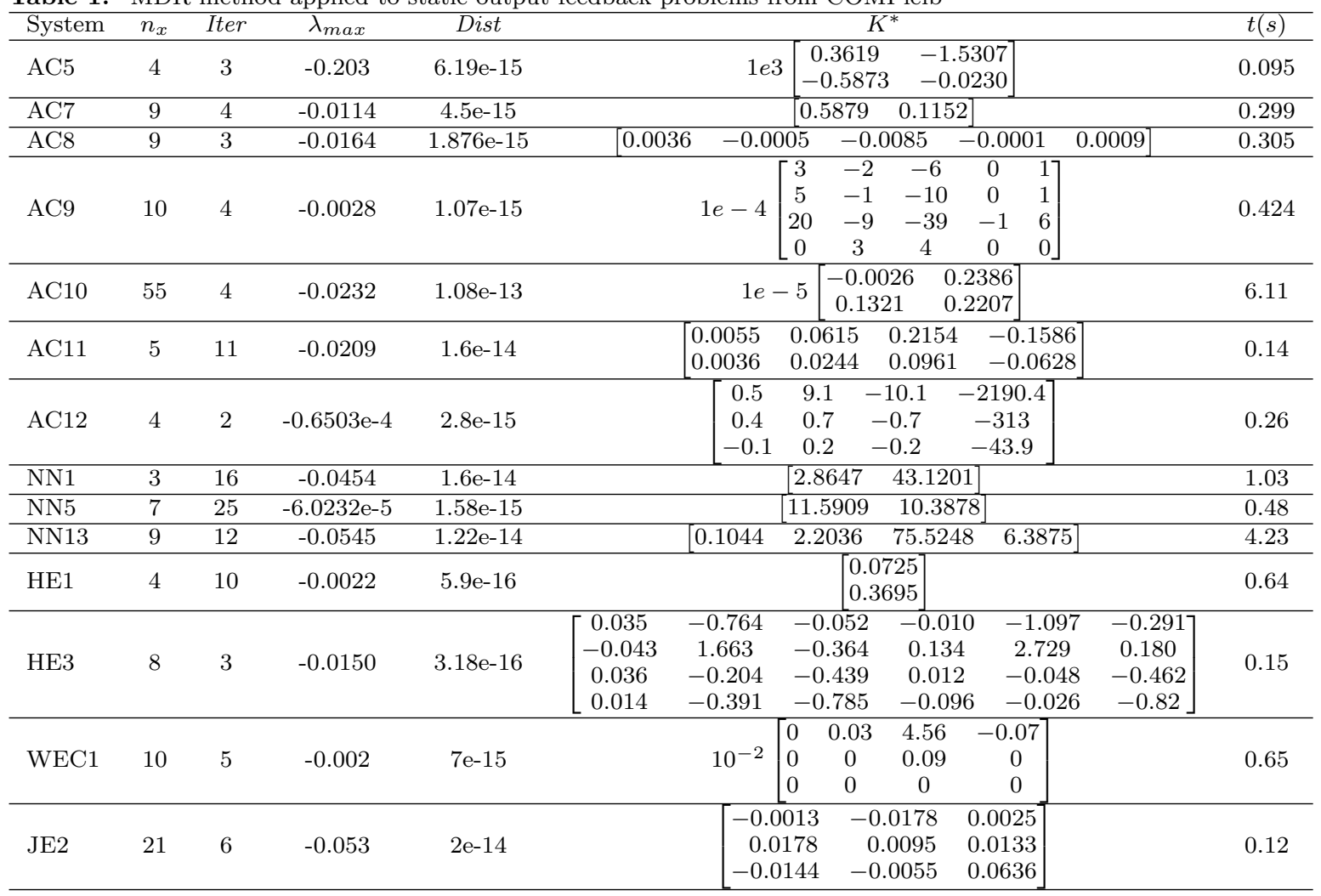

has been imposed we obtain, after 2 iterations, the structured stabilizing static output feedback $K_{s}^{*}$ :

$$
K_{s}^{*}=\left[\begin{array}{lll}
-0.0023 & 0 & -0.0023 \\
-0.0006 & 0 & -0.0006 \\
-0.7683 & 0 & -0.7739
\end{array}\right]
$$

with $\sigma\left(A+B K_{s}^{*} C\right)=\{-3.8330 \pm 1.0587 i,-1.5000 \pm 1.9365 i,-0.1102\}$.

The MDR method is then tested on the well-known COMPleib library by Friedemann Leibfritz, F. (2004). All the examples are run from a zero gain matrix and no random start is used. In Table I, $n_{x}$ designates the system size, Iter the size of MDR sequence, Dist the distance of $Z=A+B K^{*} C$ to the set of feasible output feedbacks $\overline{\mathbb{D}}_{L}, K^{*}$ the static controller gain solution and $t$ the computation-time in seconds.

\section{2. $H_{\infty}$ control}

Once more, the same academic example borrowed from (Rubio-Massegu et al., 2013) is considered. It consists of a LTI system given by (18) with the following complementary state-space matrices data:

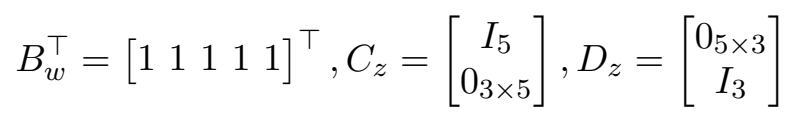


The aim is to find a stabilizing static output feedback that ensures a closed-loop $H_{\infty}$ performance less than $\gamma_{\infty}=0.78$. After 12 iterations and a computation-time of $t=1.2 s:$

$$
K_{\infty}=\left[\begin{array}{lll}
-0.7637 & 0.0572 & -0.6578 \\
-0.8107 & 0.0763 & -0.0230 \\
-1.1551 & 0.7020 & -1.2222
\end{array}\right]
$$

is obtained achieving an $H_{\infty}$ closed-loop performance of $\left\|T_{z w}(s)\right\|_{\infty}=0.7712$. Moreover, the proposed method was tested intensively on COMPleib systems. For the AC5 system, for instance, 10 iterations and a computation time of $t=0.778 \mathrm{~s}$, were required to obtain the following static output feedback gain :

$$
K_{\infty}=1 e 6\left[\begin{array}{rr}
-6.5008 & -0.1737 \\
-3.6406 & 1.9473
\end{array}\right]
$$

achieving $\left\|T_{z w}(s)\right\|_{\infty}=667.29$ which is better than the one found by HIFOO approach $\left(\gamma_{\infty}=669.56\right)$ (Arzelier, Georgia, Gumussoy \& Henrion, 2011). For the NN16 system, 3 iterations and a computation time of $t=0.225 \mathrm{~s}$, were required to obtain the following static output feedback gain

$$
K_{\infty}=1 e 6\left[\begin{array}{rrrr}
-0.0453 & 0.0070 & -0.0144 & 0.0032 \\
0.0069 & -0.0313 & -0.0032 & -0.0011 \\
-0.0144 & -0.0032 & -0.0453 & -0.0070 \\
0.0032 & -0.0011 & -0.0069 & -0.0313
\end{array}\right]
$$

achieving $\left\|T_{z w}(s)\right\|_{\infty}=0.9909$ which is substantially equal to the one found using a Particle Swarm Optimization based technique $\left(\gamma_{\infty}=0.99\right)$ (Yagoubi \& Sandou, 2011).

\section{Conclusion}

A reflection-based method for feedback synthesis under information structure constraints is presented. The approach relies on a prominent property of Hurwitz-stable matrices set and a Douglas-Rachford's like reflection optimization method. The convergence of the generated iterates continuous limit is connected to a continuous dynamical system trajectory. Hence, the Lyapunov stability theory is used to prove the local convergence of the proposed scheme. Finally, the efficiency of the proposed approach is demonstrated through several examples.

\section{References}

Arzelier, D. and Georgia, D., Gumussoy, S. and Henrion, D.(2011). $\mathcal{H}_{2}$ for Hifoo, International Conference on Control and Optimization with Industrial Applications (COIA 2011).

Bauschke, H. H. and Moursi, W.M. (2017). On the Douglas-Rachford algorithm, Mathematical Programming, 164(1), 263-284.

Bauschke, H. H., Cruz, J. B., Nghia, T. T., Phan, H. M. and Wang, X. (2014). The rate of linear convergence of the Douglas-Rachford algorithm for subspaces is the cosine of the Friedrichs angle, Journal Approximation Theory, 185, 63-79. 
Bauschke, H. H., Combettes, P. L. and Luke, D. R. (2004). Finding best approximation pairs relative to two closed convex sets in Hilbert spaces, Journal Approximation Theory, 127(2), 178-192.

Borwein, J.,M. and Sims,B. (2011). The Douglas-Rachford algorithm in the absence of convexity, Fixed-Point Algorithms for Inverse Problems in Science and Engineering, Springer New York, 93-109.

Boyd, S., El Ghaoui, L., Feron, E. and Balakrishnan, V. (1994). Linear matrix inequalities in systems and control theory, SIAM Studies in Applied Mathematics, Philadelphia, Pennsylvania.

Duan, G. R. and Patton, R. J. (1998). A note on Hurwitz stability of matrices, Automatica, $34(4), 509-511$.

Fält, M. and Giselsson, P. (2017). Optimal convergence rates for generalized alternating projections, IEEE 56th Annual Conference on Decision and Control (CDC), 2268-2274.

Franca, G., Robinson, D. P. and Vidal, R. (2018). ADMM and accelerated ADMM as continous dynamical systems, Proceedings of the 35th International Conference on Machine Learning, Stockholm, Sweden, 80,201.

Higham, N. J. (1988). Computing a nearest symmetric positive semi-definite matrix, Linear Algebra and its Applications, 103, 103-118.

Jovovic, I. V. and Malesevic, B. J. (2014). A note on solutions of the matrix equation $A X B=$ C. Scientific Publications of the State University of Novi Pazar, Ser A: Appl. Math. Inform. And Mech, 6, 45-55.

Inoue, M., Wada, T., Ikeda, M. and Uezato, E. (2015). State-space $H_{\infty}$ controller design for descriptor systems, Automatica, 59, 164 - 170.

Leibfritz, F. (2004). COMPleib: COnstrained Matrix optimization Problem library a collection of test examples for nonlinear semidefinite programs, control system design and related problems. Technical report. University of Trier, Dept. of Mathematics.

Lewis, A. S. (2007). Nonsmooth optimization and robust control, Annual Review in Control, $31(2), 167-177$.

Lindstrom, S. B. and Sims, B. (2018). Survey: Sixty years of Douglas-Rachford, Journal of the Australian Mathematical Society, 1-38.

Moreau, J. J. (1965). Proximité et dualité dans un espace hilbertien, Bulletin de la Société Mathématique, France, 95, 273-299.

Rubio-Massegu, J., Rossell, J. M., Karimi, H. R. and Palacios-Quinonero, F. (2013). Static output-feedback control under information structure constraints, Automatica, 49(1), 313316.

Ryu, E.K. and Boyd, S. (2016). A primer on monotone operator methods survey, Applied and computational mathematics, $15(1), 3-43$.

Sadabadi, M. S. and Peaucelle, D. (2016). From static output feedback to structured robust static output feedback: A survey, Annual Reviews in Control, 42, 11-26.

Scherer, C., Gahinet, P. and Chilali, M. (1997), Multiobjective output-feedback control via LMI optimization, IEEE Transactions on Automatic Control, 42, (7), 896-911.

$\mathrm{Su}$, W., Boyd, S. and Candes, E. J. (2016). A differential equation for modeling Nesterov's accelerated gradient method: theory and insights. Journal of Machine Learning Research, $17(1), 5312-5354$.

VanAntwerp, J. G. and Braatz, R. D. (2000). A tutorial on linear and bilinear matrix inequalities, Journal of Process Control, 10(4), 363-385.

Yagoubi, M. and Sandou, G. (2011). Particle Swarm Optimization for the static $\mathcal{H}_{\infty}$ static output feedback, Proceedings of the IFAC World Congress, $44(1), 12581-12586$. 